\title{
El discurso científico en la etapa de infantil en el contexto del huerto ecológico escolar
}

\author{
Lourdes Aragón iD \\ Departamento de Didáctica, Facultad de Ciencias de la Educación. Universidad de Cádiz. Cádiz. España. \\ lourdes.aragon@uca.es \\ Susana Sánchez \\ Departamento de Didáctica de la Lengua y la Literatura, Facultad de Ciencias de la Educación. Universidad \\ de Cádir. Cádir. España.susana.sanchez@uca.es
}

\author{
Juan Manuel Enríquez \\ CEIP Reyes Católicos de Cádiæ, Cádiæ, España.jmenriquez.ecp.hbvs@gmail.com
}

[Recibido: 24 enero 2020. Revisado: 1 junio 2020. Aceptado: 9 Julio 2020]

\begin{abstract}
Resumen: Desde el compromiso de promover la alfabetización científica desde edades tempranas, la siguiente investigación tiene como objetivo explorar el funcionamiento del discurso científico de niños y niñas de 5 años en momentos de asamblea y en el marco de una secuencia didáctica contextualizada en el huerto ecológico escolar. Para ello, a partir de las transcripciones de cuatro asambleas se ha establecido un sistema de categorías emergentes, aplicado sobre los datos y consensuado entre expertos. Los resultados muestran que los niños de 5 años aportan datos e información al discurso (31,6\%), aportan esquemas de conocimiento (39,8\%), argumentan sobre los esquemas que comparten (24\%) y en menor medida, hacen emerger modelos iniciales explicativos $(4,6 \%)$. Respecto a los esquemas de conocimiento que surgen, estos giran en torno a los elementos necesarios en el huerto (luz, tierra, agua, profundidad), y permiten visualizar e inferir los modelos iniciales explicativos que maneja el alumnado a estas edades. En conclusión, los resultados arrojan evidencias respecto de la capacidad de desarrollar la competencia científica desde edades tempranas y la idoneidad del uso del huerto ecológico escolar como contexto para la promoción de habilidades cognitivo lingǘsticas que influyen en la construcción del conocimiento científico.
\end{abstract}

Palabras clave: Asamblea de aula; Huerto ecológico; Discurso científico en aula; Educación Infantil; Esquemas de conocimiento; Modelos

\section{Scientific discourse in Early Childhood Education in the context of the Ecological School Garden}

\begin{abstract}
According to the necessity to develop scientific literacy from early ages, the aim of this work is to explore scientific discourse of 5 years-old children at circle time during a didactic sequence related to their ecological school garden. To get those achievements, an emergent-cathegories system has been stablished by the analysis of transcriptions obtained from four circle time registrations, being this system agreed by experts. Results show that 5 years-old children contributed with data and information to the discourse (in a 31,6\% of the cases), they supply knowledge schemata (in a 39,8\% of the cases), they offer arguments on the shared schemata (in a $24 \%$ of the cases) and, in a less measure, they build initial explaning models (in a 4,6\% of the cases). In relation to knowledge schemata that are built in this context, they are about the necessary elements in the garden (light, earth, water, soil or depth), and they allow to visualize and to infere the initial explaining models handled by the children at that age. In conclusion, results show evidences respect to the capacity of developing scientific competence from early years as well as the suitability of the use of ecological school garden as a context to promote cognitive-linguistic skills which influence on scientific knowledge construction.
\end{abstract}

Keywords: Circle time; Ecological school garden; Scientific classroom discourse; Early childhood education; Knowledge schemata; Models

Para citar este artículo: Aragón L., Sánchez S. y Enríquez J.M. (2021) El discurso científico en la etapa de infantil en el contexto del huerto ecológico escolar. Revista Eureka sobre Enseñanza y Divulgación de las Ciencias 18(1), 1103 doi: 10.25267/Rev_Eureka_ensen_divulg_cienc.2021.v18.i1.1103 


\section{Introducción}

La enseñanza de las ciencias busca educar en contenidos de ciencia y tecnología de especial relevancia para quien estudia desde un enfoque integral, sistémico e interdisciplinar (AsencioCabot 2017). Esta visión supera una finalidad puramente propedéutica, cuyo objetivo sería el acceso a estudios científicos superiores. Si partimos de la idea de que la ciencia escolar es altamente relevante para formar una ciudadanía crítica, capaz de tomar decisiones en asuntos sociocientíficos y hacer frente a los problemas y realidades sociales, culturales y ambientales, la principal finalidad educativa de la enseñanza de las ciencias debe ser promover la alfabetización científica de los futuros ciudadanos y ciudadanas (Acevedo 2004). De hecho, una educación científica y tecnológica se vuelve imprescindible (Pujol 2003) y se conecta con la alfabetización lingüística en su nivel epistémico (Wells 1987). Esto conlleva tomar decisiones curriculares y metodológicas adecuadas, como abordar contenidos de ciencias vinculados a su utilidad para la persona y la sociedad en la que vive (Marín 2005). Este planteamiento resulta complejo por diversas cuestiones. Por un lado, según Pontes, Poyato y Oliva (2017), la población se expone a un continuo aluvión de información que los medios de comunicación suelen mostrar de manera simplista y poco o nada contrastada. Se añade a esto la presencia de movimientos pseudocientíficos contrarios a los planteamientos que se pretenden construir en las aulas. Además, encontramos diseños curriculares alejados de la práctica social de la ciencia, sistemas de evaluación determinados por un enfoque propedéutico o un profesorado poco comprometido con el sentido social del conocimiento científico (Morillo Moronta 2008) y su articulación comunicativa (Jorba, Gómez y Prat 2000).

A pesar de estas dificultades, el objetivo es que los y las escolares desarrollen su competencia científica que, según Pedrinaci et al. (2012 p.5) es "un conjunto integrado de capacidades personales para utilizar el conocimiento científico con el objetivo de describir, predecir y explicar, pero también, para comprender las características propias de la ciencia, esto es, formular e investigar hipótesis, documentarse, argumentar y tomar decisiones en torno a diversas cuestiones tecnocientíficas". Este proceso es además gradual y en él interviene el conocimiento sobre las ciencias y las actitudes hacia ellas (Hodson 1994).

Por otra parte, la competencia científica contempla tres prácticas estrechamente relacionadas entre sí: la indagación, la modelización y la argumentación (Jiménez-Aleixandre, Bravo y Puig 2009; Jiménez-Aleixandre y Crujeiras-Pérez 2017). La indagación es entendida como la transposición didáctica de la investigación científica. En el ámbito escolar suele concretarse en contextos de investigación que propician procedimientos científicos como observaciones, formulación de hipótesis, experimentos, etc., en los que los y las estudiantes se plantean preguntas y obtienen sus propios datos (Couso 2014). La modelización, también planteada desde un contexto de ciencia escolar, se entiende como un proceso de aprendizaje que conlleva construir modelos, aplicarlos, revisarlos, modificarlos e incluso sustituir unos por otros (Justi y Gilbert 2002). Finalmente, la argumentación, según Jiménez-Aleixandre y Puig (2010), es evaluar un conocimiento a partir de pruebas disponibles. La capacidad de argumentar permite así conectar explicaciones y pruebas para evaluar enunciados, teorías o modelos. Estas prácticas científicas entendemos que deberían iniciarse desde etapas tempranas como se contempla en el Informe ENCIENDE (COSCE 2011) y como sostienen, entre otros, Acevedo, Vázquez y Manassero (2003).

Son escasos los estudios que existen para el nivel de Educación Infantil (desde ahora, EI), sin embargo, sí disponemos de trabajos que evidencian la capacidad de niños y niñas de 3-4 años de comunicar y expresar, a través de distintos tipos de representaciones, sus propios modelos construidos en torno a contenidos de ciencias (Monteira y Jiménez-Aleixandre 2019). Mazas, Gil-Quílez y Martínez-Peña (2018), por su parte, constatan la posibilidad de trabajar en estas 
primeras etapas a partir de proyectos que nacen de la observación del entorno y que favorecen la construcción de modelos parciales de los fenómenos observados (Monteira y JiménezAleixandre 2016; Tunnicliffe y Reiss 2000). Por ello, resulta relevante reflexionar sobre cómo el contexto escolar y, en particular, el discurso compartido que se genera en las aulas, puede fomentar la competencia científica desde edades tempranas.

\section{El discurso científico en Educación Infantil}

Ser competente en ciencia supone ser capaz de hablar, leer y escribir ciencia (Lemke 1997; Sanmartí 2007), es decir, generar y comprender discursos sobre los que se articula un conocimiento validado. El discurso científico canaliza por tanto los procesos de proponer información, problematizarla, discutir ideas y argumentar en función de evidencias y establecer conclusiones. Es un discurso racional, de carácter progresivo, ya que está siempre abierto a la refutación y el avance, y es también exigente, tanto en lo que respecta a la precisión de las realidades a las que alude como en lo relativo a su propio funcionamiento discursivo. En este sentido, Lemke (1997) distingue entre el patrón temático (los contenidos que se abordan en el discurso) y el patrón estructural (la organización de dicho discurso), con el fin de mostrar esta doble exigencia en el desarrollo de la competencia científica.

Los niños y niñas de EI inician su capacidad de comunicarse verbalmente; su competencia comunicativa se caracteriza por ser experimental y creativa, en paulatina adaptación hacia lo convencional. Esto no impide cumplir las diversas funciones comunicativas que la vida escolar y cotidiana les demanda; así, toman decisiones en grupo, expresan sentimientos complejos, plantean hipótesis, argumentan soluciones y afrontan otros muchos procesos comunicativos que necesitan llevar a cabo en función de la actividad que realizan en sociedad (Domínguez y Barrio 2001; Ruiz Bikandi 2002; Mercier 2011; Migdalek, Rosemberg y Santibáñez 2015; Sánchez 2016; Mérida, González y Alfaya 2017).

Sin embargo, se ha constatado que en EI el profesorado no suele verbalizar las observaciones de los fenómenos naturales, y escasean actividades propias de la metodología científica (Cantó Doménech, de Pro Bueno y Solbes 2016), a pesar de que es posible favorecer y potenciar la construcción de modelos mentales iniciales sobre contenidos científicos a través de la observación de fenómenos naturales cotidianos, como punto inicial para fomentar la comunicación y verbalización de las ideas del alumnado (Mazas et al. 2018). Existen pocos trabajos que aborden el discurso científico en aulas de EI en nuestro entorno (por ejemplo, Ríos 2013; Monteira y Jiménez-Aleixandre 2016; García-González y Schenetti 2019; Monteira, Jiménez-Aleixandre y Siry 2020). Sin embargo, su interés es innegable para el desarrollo de la competencia científica y de la comunicación oral formal (Sánchez 2016).

Los trabajos que existen en torno a los modelos mentales que presentan niños y niñas de edades tempranas sobre contenidos concretos son muy limitados. En este estudio, manejan esquemas previos mentales referente principalmente al ciclo del agua y a las plantas. Algunas investigaciones constatan que a los 4 y 5 años niños y niñas manejan modelos mentales muy iniciales respecto a estos contenidos. A estas edades predomina un modelo de ser vivo en el que solo consideran a los animales pero no a las plantas, utilizando como principal criterio la ausencia de movimiento. Así, las plantas no son consideradas seres vivos debido a su naturaleza aparentemente estática (Garrido 2007; Tunnicliffe 2020; Villanueva 2017). Otros estudios revelan que el alumnado de estas edades maneja ideas sencillas sobre la estructura y la función de las plantas. Anderson, Ellis, Jones (2014) indican que un bajo porcentaje del alumnado de 5 años representó hojas sin incluir raíces. Por otro, el modelo de planta que parece predominar en este momento es la planta tipo flor (Anderson et al. 2014; McNair y Stein 2001). Respecto al contenido del ciclo del agua, Mazas et al. (2018) evidencian que a los 3 
años manejan esquemas previos en los que el agua sufre cambios y transformaciones cuando se someten a diferentes situaciones (cambios de temperatura), y contemplan la idea de que el agua está en distintos lugares y estados en la naturaleza. En niños y niñas de edades más avanzadas, parece predominar un modelo no cíclico del agua, en el que no se considera el cierre entre las entradas y las salidas de agua, y por tanto, la circulación de agua queda interrumpida en algún momento (Gutiérrez 1998; Márquez y Bach 2007).

\section{El huerto ecológico escolar: recurso y contexto de aprendizaje de las ciencias}

En los últimos años se ha incrementado significativamente el uso de huertos escolares como recurso didáctico. Esta proliferación se asocia al enorme potencial didáctico que presenta este recurso (Escutia 2009). El huerto no solo se vincula a conocimientos específicos de ciencias (Eugenio y Aragón, 2016), también se conecta con temas transversales como la educación para la sostenibilidad o la educación para la salud y la alimentación, sobre todo con el consumo de verduras y hortalizas escolares pueden mejorar el aprendizaje de los estudiantes o ayudar a estos a crear hábitos de alimentación más saludables (Grimm-Rice 2011). En algunos casos, el Huerto Ecológico Escolar (HEE en adelante) se emplea como eje central para plantear nuevos modelos didácticos y poder hacer frente a los desafíos ambientales que la sociedad se enfrenta; bien a través de la perspectiva del decrecimiento (Rodríguez-Marín, Fernández-Arroyo y García 2015), y desde un enfoque de una educación para el desarrollo sostenible, siendo un elemento clave dentro de un marco emergente como es la agroecología escolar, una disciplina que conlleva una práctica centrada en el sistema alimentario escolar con una fuerte implicación de todos los actores de la comunidad educativa y orientado a la transformación social y ciudadana desde la etapa de infantil (Egea-Fernández, Egea-Sánchez y Guerreo 2016; Llerena y Espinet 2017). El HEE se utiliza para trabajar valores con la idea de buscar alternativas a modelos de producción y consumo actuales (Cáceres 2012), los niños y las niñas toman conciencia de los ritmos de la naturaleza, desarrollan el sentido de la implicación, colaboración, y responsabilidad, y el compromiso del alumnado a la hora de gestionar el huerto (Marín 2005).

Actualmente existen numerosas publicaciones que documentan experiencias y propuestas didácticas llevadas a cabo en torno al uso HEE, sin embargo, la mayoría de ellas se centran en explicar y describir cómo estas se llevan a cabo. Creemos que la investigación puede aportar aún mucho sobre el huerto como contexto de aprendizaje y como espacio para favorecer el desarrollo de la competencia científica en su doble plano conceptual y comunicativo. Con la siguiente investigación nos planteamos un doble objetivo: 1) explorar el funcionamiento del discurso científico en un aula del alumnado de 5 años en los momentos de habla compartida en la asamblea de clase en el marco de una secuencia didáctica (SD en adelante) dirigida al diseño y puesta en marcha de un HEE y 2) identificar los esquemas de conocimiento que afloran durante dicho discurso, cómo estos se movilizan y permiten visualizar modelos mentales presentes a estas edades.

En el contexto específico de estudio, se decidió diseñar y construir un huerto ecológico tipo bancal, de madera, elevado y con ruedas de dimensiones de 290x90 cm, dado el escaso espacio disponible en el patio del centro. Para su preparación y siembra participaron activamente el alumnado de las cuatro aulas de 4 y 5 años junto a sus tutoras. Para ello, se utilizaron técnicas propias de permacultura como el uso de humus de lombriz, la técnica del acolchado y la asociación entre plantas. 


\section{Metodología}

\section{Contexto de investigación y participantes}

Este estudio se enmarca en un proyecto de investigación concedido por la Junta de Andalucía (PIV-040/17) durante el curso 2017/2018 y cuyo objetivo es evaluar el uso del HEE como contexto de aprendizaje para el desarrollo de la competencia científica en EI. El trabajo de campo duró cuatro meses, desde febrero hasta mayo del 2018. Se trata de un estudio cualitativo, de corte naturalista y próximo al estudio de caso, como método para aproximarnos y entender de una manera más holística un fenómeno social complejo como puede ser la realidad de un aula (Palou y Fons 2013).

El caso de estudio se trata de un grupo clase de 25 niños y niñas de 5 años de edad (15 niños y 10 niñas) y su tutor, pertenecientes al CEIP Reyes Católicos de Cádiz. El maestro es un docente con más de 16 años de experiencia en EI y coautor de este trabajo. Se diseñó e implementó una SD en torno al HEE con el objeto de contribuir al desarrollo de la competencia científica del alumnado de 4 y 5 años. La organización de la SD alterna actividades individuales, de pequeño grupo y de gran grupo, con duración variable, como se detalla en el anexo 1. Algunas actividades se realizaron a lo largo de varias sesiones con una duración breve pero continuada en el tiempo (actividad 14 y 15). Otras se realizaron en una sesión de 45 minutos máximo (actividades 2, 5 y 7). Para este trabajo se han seleccionado las actividades 1, 4, 12 y 14, cuatro sesiones de asamblea en gran grupo, de duración de 25, 45, 22 y 41 minutos, respectivamente.

\section{Instrumentos para la recogida de información}

Las asambleas se plantearon como espacios de comunicación orientados a la construcción conjunta del discurso por parte de niñas y niños bajo la moderación del docente. La asamblea inicial tuvo como objetivo explorar las ideas previas del alumnado sobre el HEE y en este caso se dejó total libertad al docente para gestionar la asamblea. La segunda asamblea se dirigió a promover la argumentación en función de las ideas iniciales surgidas en el grupo durante la primera asamblea. Para ello, las investigadoras propusieron al docente elaborar junto a niños y niñas una lista de cosas necesarias para tener un huerto y posteriormente establecer un orden de importancia de esos elementos consensuado en el grupo-clase. Se buscaba así orientar el discurso infantil hacia la explicación-argumentación científica vinculada a sus conocimientos previos sobre los elementos presentes en un huerto y promover la justificación en sus respuestas. Las ideas de niños y niñas y el orden de los elementos quedaron registrados en un mural.

La tercera y cuarta asamblea tuvieron como objetivo favorecer el discurso científico a partir de la observación de los fenómenos ocurridos en dos experiencias prácticas realizadas antes de cada una de las asambleas. La primera de ellas (actividad 12 de la SD) consistió en colocar en un semillero dos tipos de semillas distintas. Cada grupo puso un poco de tierra en los semilleros, colocaron las semillas, taparon con tierra y finalmente, regaron y etiquetaron. Transcurrida una semana se llevó a cabo la tercera asamblea para discutir y argumentar sobre los hechos ocurridos y observados tras la siembra. La segunda actividad práctica (actividad 14 de la SD) se planteó a partir de una pregunta problema inicial: ¿qué necesitan las semillas para nacer? Para lo cual, cada equipo tuvo que identificar y separar los diferentes tipos de semillas y no semillas de una bolsa y verbalizar qué necesitaba una semilla para nacer. A continuación, el docente proporcionó a los grupos los materiales para llevar a cabo la experiencia en función de las respuestas dadas a la pregunta problema. Cada grupo se ocupó de sembrar sus semillas según las distintas variables: 1) semillas sembradas con luz y sin luz; 2) semillas sembradas con agua y sin agua; 3) semillas en tierra, sin tierra y en algodón y 4) semillas sembradas a diferente 
profundidad. Transcurridas dos semanas se realizó la cuarta asamblea, dirigida a discutir y argumentar sobre los fenómenos ocurridos.

Respecto a la gestión y desarrollo de las asambleas 2, 3 y 4, las investigadoras pautaron su organización previamente con el docente haciendo especial hincapié en: 1) que no provocara un orden de participación, sino que permitiera que se construyera naturalmente el discurso; 2) que comentara o ejemplificara con alguna situación concreta que ayudara a continuar con el discurso si el alumnado no intervenía; 3) que recurrieran al conocimiento que ya se había explicitado en asambleas anteriores; 4) que provocaran que los niños y niñas dijeran el porqué de sus afirmaciones; 5) que dejaran un breve tiempo de silencio para dejarles pensar o al menos que ralentizaran la formulación de cada pregunta, dirigiendo la mirada al grupo-clase.

En el caso de las asambleas 3 y 4, que se basaban en experiencias concretas, se indicó además que: 1) el docente recordara los experimentos realizados y retomara para el grupo ideas ya comentadas; 2) que planteara preguntas mediadoras para poder observar cambios; y 3) si aparecían razonamientos poco científicos pero sí lógicos, plantearles la posibilidad de dudar de dichos razonamientos y proyectar con ellos y ellas cómo sería posible comprobar las cuestiones que planteaban. Cada una de las asambleas fue grabada en audio y vídeo por las investigadoras y posteriormente transcrita. Se eliminaron algunos fragmentos ajenos al discurso común.

\section{Análisis de datos}

El discurso de cada asamblea fue transcrito siguiendo el procedimientos de análisis del discurso en contextos de aula: se dividió en secuencias definidas por su coherencia semántica y en intercambios determinados por la estructura de participación (Sánchez, 2010). A partir de aquí, se realizó un análisis en dos niveles. En primer lugar, la interacción en cada intercambio se analizó mediante procedimientos específicamente adaptados al contexto de esta investigación, esto es, a la comprensión de los procedimientos de construcción conjunta de un discurso científico en EI o patrón estructural según Lemke (2001). En este trabajo nos hemos centrado en las intervenciones de niños y niñas considerándolas como aportaciones al discurso compartido; para ello, se exploraron dichas aportaciones considerando cuatro dimensiones emergentes: aportación de información, establecimiento de esquemas de conocimiento y argumentación sobre los esquemas de conocimiento y modelización (Tabla 1). Entendemos como aportar información los movimientos del discurso en que los niños y niñas ofrecen datos aislados, describen, explican, retiran información, generan preguntas, pero siempre desde un plano conceptual más superficial. Por otro lado, hablamos de esquemas de conocimiento cuando expresan (aportan, aceptan o rechazan) sus modelos mentales, entendidos como representaciones internas y personales, cuya finalidad es explicar, predecir y describir fenómenos del mundo que nos rodea (Greca y Moreira 2000). Hemos diferenciado entre la aportación de esquemas de conocimiento y la argumentación sobre ellos, que añade nueva información para sostener, especificar o refutar dichos esquemas. Finalmente, se establece la categoría modelización, teniendo en cuenta que la construcción de modelos en educación infantil no consiste en adquirir el modelo en sí, sino de elementos clave que conforman el modelo científico. Son los denominados modelos precursores o modelos iniciales, que poseen un rango de aplicación limitado y que solo permiten establecer relaciones causa-efecto simples pero consideramos como imprescindibles a la hora de profundizar en el conocimiento de los niños y niñas (Canedo-Ibarra et al. 2012; Lorenzo Flores, Sesto Varela y García-Rodeja 2018). En este nivel de análisis, por tanto, hemos considerado las aportaciones en cuanto que acciones, sin profundizar en su contenido conceptual. 
Tabla 1. Sistema de categorías emergentes considerados para analizar el discurso científico durante las asambleas (Fuente: Elaboración propia).

\begin{tabular}{|c|c|c|c|c|}
\hline Dimensiones & $\begin{array}{l}\text { Aportación } \\
\text { información }\end{array}$ & $\begin{array}{l}\text { Aportación de } \\
\text { esquemas de } \\
\text { conocimiento }\end{array}$ & $\begin{array}{l}\text { Argumentación } \\
\text { sobre los esquemas }\end{array}$ & Modelización \\
\hline Categorías & $\begin{array}{l}\text { No sabe/no contesta } \\
\text { Aporta información } \\
\text { no relevante } \\
\text { Aporta información } \\
\text { relevante } \\
\text { Retira información } \\
\text { Contextualiza } \\
\text { Genera pregunta } \\
\text { Describe } \\
\text { Define } \\
\text { Explica }\end{array}$ & $\begin{array}{l}\text { Aporta esquema } \\
\text { Apoya esquema } \\
\text { Se opone a } \\
\text { esquema }\end{array}$ & $\begin{array}{l}\text { Aporta razones a favor } \\
\text { Apoya razones a favor } \\
\text { Aporta razones en } \\
\text { contra } \\
\text { Apoya razones en contra } \\
\text { Se opone a razones } \\
\text { Aporta condiciones a } \\
\text { favor } \\
\text { Apoya condiciones a } \\
\text { favor } \\
\text { Aporta condiciones en } \\
\text { contra an a } \\
\text { Apoya condiciones en } \\
\text { contra Se opone a } \\
\text { condiciones } \\
\text { Aporta razones a favor } \\
\text { de las condiciones } \\
\text { Aporta razones en } \\
\text { contra de } \\
\text { condiciones las } \\
\text { Busca explicaciones } \\
\text { apoyándose en esquema } \\
\text { Formula hipótesis }\end{array}$ & $\begin{array}{l}\text { Explicita modelo } \\
\text { Apoya modelo } \\
\text { Se opone a modelo } \\
\text { Aporta razones en } \\
\text { contra del modelo } \\
\text { Propone } \\
\text { diseño/experimento } \\
\text { Concluye (modeliza) } \\
\end{array}$ \\
\hline $\begin{array}{l}\text { Total de categorías } \\
\text { por dimensión }\end{array}$ & 9 & 3 & 14 & 6 \\
\hline
\end{tabular}

El segundo nivel de análisis sí se dirigió a abordar la organización conceptual que se explicita en el discurso o patrón temático (Lemke 1997). Para ello, se identificaron los esquemas de conocimiento científico que afloraron en cada secuencia de las 4 asambleas desde esta perspectiva conceptual, es decir, se consignó el orden de aparición en el discurso de cada uno de los esquemas, sin cuantificar la frecuencia de su aparición. Hemos considerado que la aparición de dichos esquemas es una contribución conceptual al discurso compartido que permite explorar los modelos iniciales que maneja el grupo-clase.

\section{Resultados y discusión}

\section{Sobre el discurso científico infantil}

En el conjunto de las cuatro asambleas se concreta el discurso científico compartido por el grupo, que se estructura de la forma que se muestra en la figura 1:

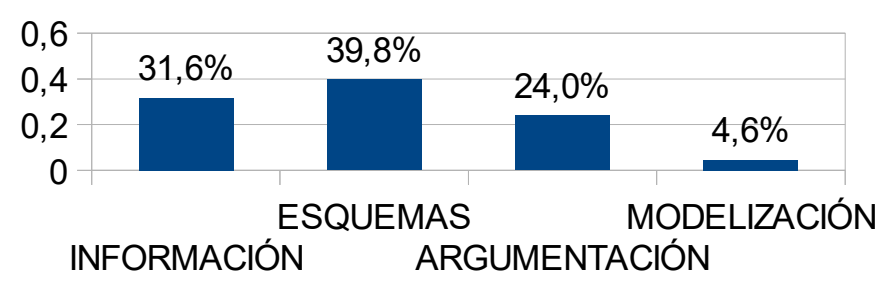

Figura 1. Estructura del discurso científico compartido presente en las cuatro asambleas. 


\section{Respecto de la aportación de la información (31,6\% del total)}

Los niños y niñas aportan datos al discurso, lo hacen de forma relevante $(35,1 \%)$, describiendo $(38,2 \%)$ y, en menor medida, explicando $(10,5 \%)$ o definiendo $(7 \%)$ la información de la que disponen. La falta de respuesta o la aportación de información no relevante al hilo del discurso son bastante bajas $(3,9 \%$ y 2,6\% respectivamente) (figura 2). Encontramos descripciones asociadas a huerto como "que crecen fresas" o definiciones como "es como un paraíso bonito".

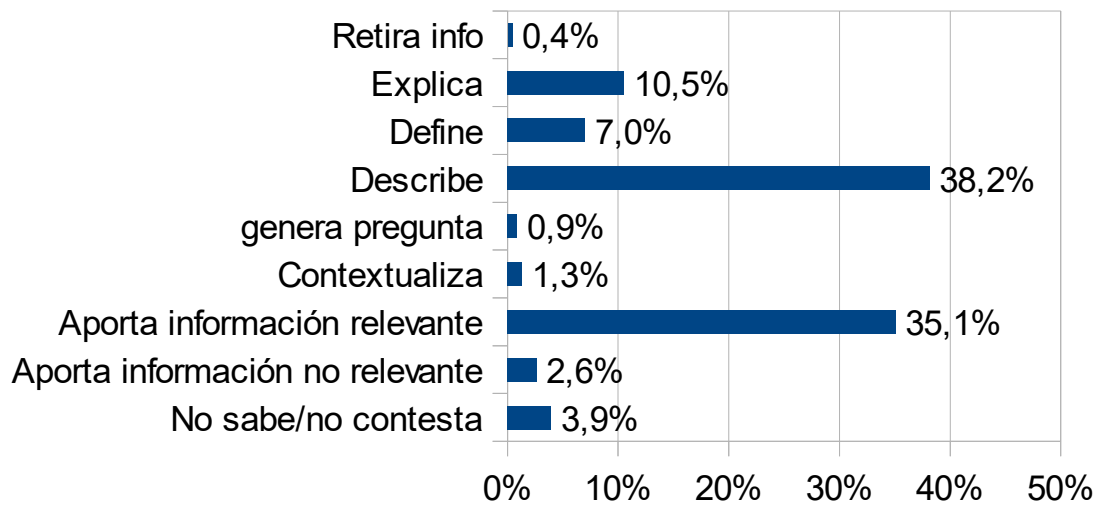

Figura 2. Porcentaje de categorías de la Dimensión aportación información.

\section{Respecto de la aportación de esquemas $(39,8 \%$ del total)}

La explicitación de un conocimiento más estructurado es la dimensión más presente en el discurso infantil. Un ejemplo de aportación de esquema es la siguiente intervención: "en un huerto, para mí, tiene las comidas, las verduras, tú las plantas ahí y cuando llega la lluvia y el sol se van creciendo". La actividad se corresponde en mayor medida (69\%) con el apoyo -por repetición de la idea- de esquemas de conocimiento aflorados previamente (sobre todo por sus iguales $(21,3 \%)$, pero también por su maestro) y es poco frecuente $(9,8 \%)$ que haya oposición a ese conocimiento, como se muestra en la figura 3 :

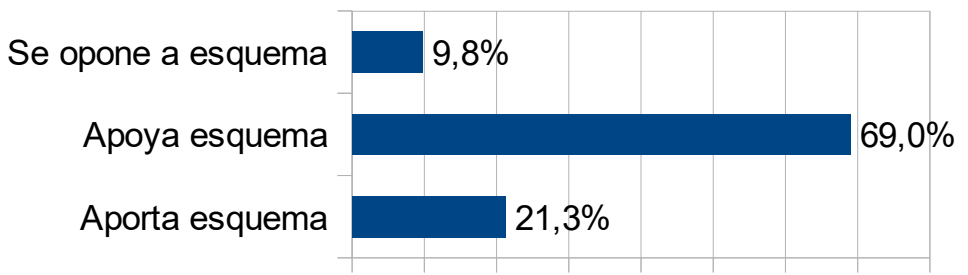

0\% 10\% 20\% 30\% 40\% 50\% 60\% 70\% 80\%

Figura 3. Porcentaje de las diferentes categorías de la Dimensión aportación esquemas

\section{Respecto de la argumentación sobre los esquemas (24\% del total)}

Las opciones de participación en el discurso se diversifican aquí puesto que se orientan a razonar a favor o en contra de los esquemas de conocimiento que han aflorado, o bien a restringir su valor de verdad a través de condiciones que a su vez se aceptan o se excluyen (figura 4). Niñas y niños intervienen más para aportar razones que apoyan los esquemas dados $(33,5 \%)$, para apoyar condiciones que restringen el valor de verdad $(20,2 \%)$ y, en menor medida, para aportar dichas condiciones (13,3\%). Es interesante cómo, aparte de hacerles aceptar, condicionar, refutar los esquemas de conocimiento que manejan, la actividad discursiva les lleva a buscar explicaciones a partir de los esquemas $(8,7 \%)$ o a formular 
hipótesis $(6,4 \%)$. La siguiente aportación es un ejemplo de cómo se explicitan condiciones que restringen los esquemas: para el esquema (3) el buerto está cerca de casa y se riega, el grupo restringe cuánto se ha de regar con aportaciones como: "todos los días no"; "no, porque si no, se pueden ahogar"; "¡claro! Un día un poquito, otro día un poquito".

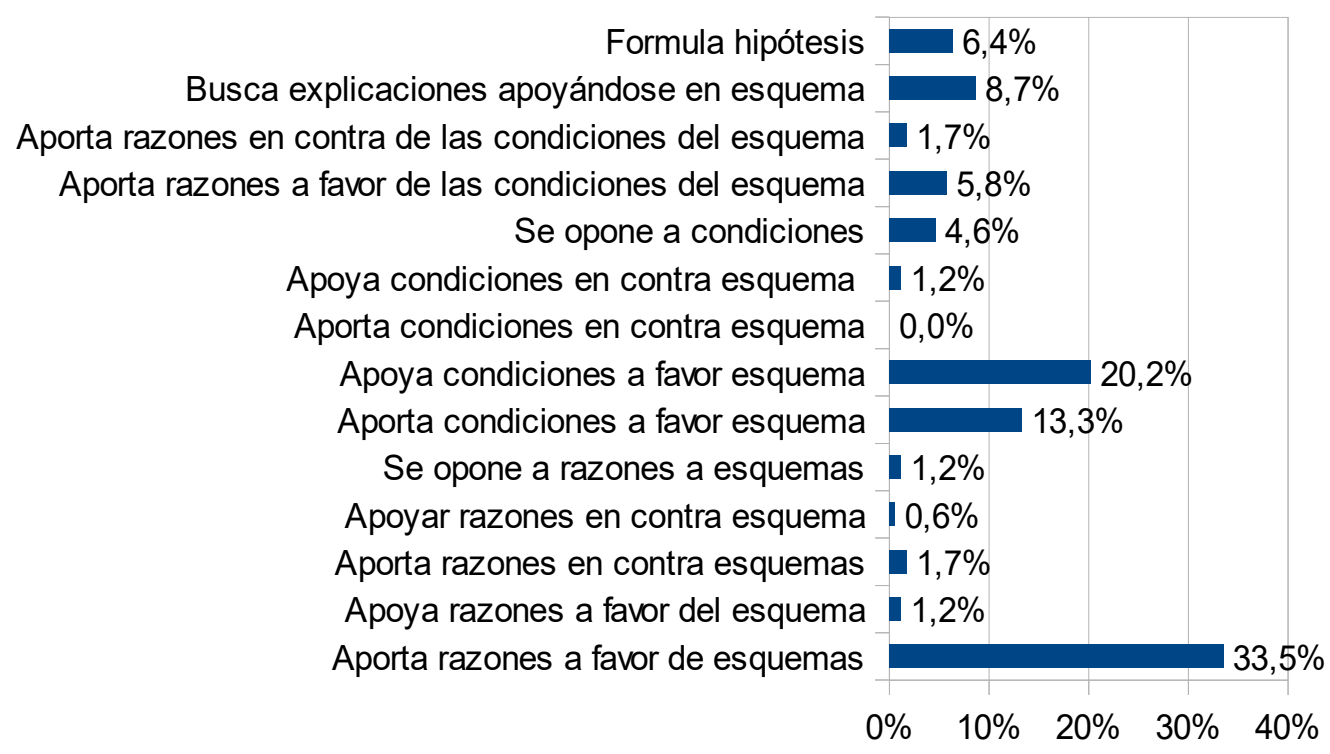

Figura 4. Porcentaje de las diferentes categorías de la Dimensión argumentación sobre esquemas

\section{Respecto de la modelización $(4,6 \%$ del total)}

Esta dimensión es la de menor presencia en nuestros datos. Cuando aparece, se corresponde en un 54,5\% con el establecimiento de conclusiones sobre los esquemas mentales que han aflorado y evolucionado en el discurso compartido y que de este modo les permiten establecer modelos científicos iniciales (figura 5). Un ejemplo de conclusión es el siguiente: "el agua es importante para las plantas y para las personas". Unido a esa capacidad de modelizar encontramos intervenciones que apoyan esos modelos por repetición de ideas. De forma más minoritaria, los niños y las niñas llegan a plantear experimentos que profundizarían en dichos modelos iniciales $(12,1 \%)$ o los explicitan $(6,1 \%)$.

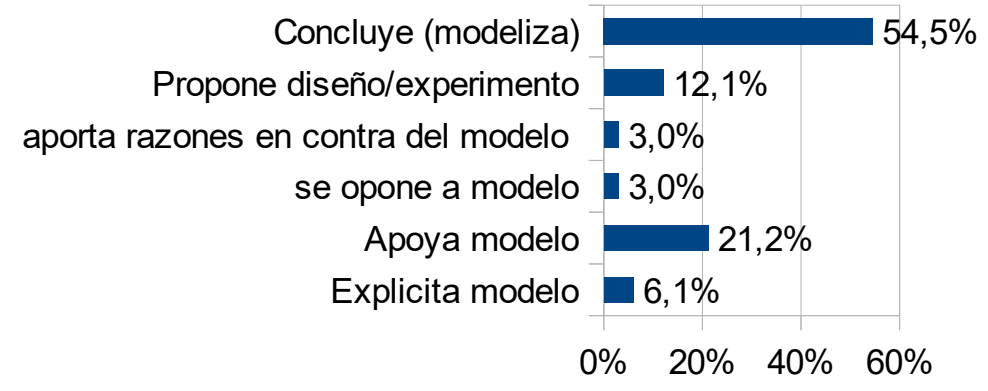

Figura 5. Porcentaje de categorías de la dimensión de modelización. 


\section{Sobre los esquemas mentales y modelos científicos iniciales presentes en el discurso}

Los esquemas permiten visualizar el modelo inicial mental que aflora en ese momento. Los esquemas son estructuras dinámicas, que surgen a lo largo del discurso científico y que son aceptadas, rechazadas, e incluso retomadas entre asambleas. Existe por tanto un movimiento de esquemas, que se conectan entre sí, y que permiten vislumbrar modelos precursores explicativos presentes en niños y niñas de 5 años. Durante la asamblea 1, se identificaron un total de 7 esquemas que permiten aflorar dos modelos iniciales: modelo de huerto y el modelo de las plantas como seres vivos. En la asamblea 2 se identificaron un mayor número de esquemas que en la asamblea 1 (22 esquemas en total). En la secuencia 3 de la asamblea 2, por ejemplo, se evidencia cómo los esquemas se complementan unos con otros, y se conectan con otros esquemas, pero también con modelos explicativos que se activan previamente. Así, se explicita el modelo de buerto que mantienen los niños, este es el buerto como lugar, más o menos extenso, el cual se conecta con otro esquema que aparece en la secuencia 2: un buerto está en un campo. En esta asamblea emergen nuevamente el modelo explicativo las plantas como seres vivos, pero también el modelo del ciclo del agua (Tabla 2).

Tabla 2. Esquemas y modelos explicativos emergentes identificados en las asambleas 1 y 2 (Fuente: Elaboración propia).

\begin{tabular}{|c|c|c|c|c|}
\hline Asamblea & $\begin{array}{l}\mathbf{N}^{\mathbf{o}} \quad \text { Total } \\
\text { Esquemas }\end{array}$ & Secuencia & Esquemas de conocimiento & $\begin{array}{l}\text { Modelos } \\
\text { iniciales }\end{array}$ \\
\hline \multirow[t]{3}{*}{1} & 2 & $\begin{array}{l}\text { 1) ¿Qué son los } \\
\text { huertos? }\end{array}$ & $\begin{array}{l}\text { (1) Es un lugar donde se cultivan } \\
\text { verduras, están presentes las plantas, el } \\
\text { agua y el sol. } \\
\text { (2) Es un lugar donde crecen las verduras. }\end{array}$ & Modelo de buerto \\
\hline & 3 & $\begin{array}{l}\text { 2) ¿Cómo son los } \\
\text { huertos qué } \\
\text { conocemos? }\end{array}$ & $\begin{array}{l}\text { (3) Un huerto está al lado de casa y se } \\
\text { riega. } \\
\text { (4) En un huerto hay aceitunas } \\
\text { (5) Las aceitunas crecen en los arboles, no } \\
\text { en los huertos. }\end{array}$ & Modelo de buerto \\
\hline & 2 & $\begin{array}{l}\text { 3) ¿Qué se puede } \\
\text { hacer en un } \\
\text { huerto? }\end{array}$ & $\begin{array}{l}\text { (6) Hay que esperar para recoger los } \\
\text { productos del huerto. } \\
\text { (7) Hay que regar en el huerto para que } \\
\text { pueda producir. }\end{array}$ & $\begin{array}{l}\text { Modelo las } \\
\text { plantas son seres } \\
\text { vivos }\end{array}$ \\
\hline \multirow[t]{4}{*}{2} & 6 & $\begin{array}{l}\text { 2) ¿Qué hay en un } \\
\text { huerto? }\end{array}$ & $\begin{array}{l}\text { (1) plantas, verduras y flores. } \\
\text { (2) animales } \\
\text { (3) está en el campo } \\
\text { (4) tierra } \\
\text { (5) agua } \\
\text { (6) sol }\end{array}$ & $\begin{array}{l}\text { Modelo las } \\
\text { plantas son seres } \\
\text { vivos } \\
\text { Modelo del ciclo } \\
\text { del agua } \\
\text { Modelo de buerto }\end{array}$ \\
\hline & 5 & $\begin{array}{l}\text { 3) ¿Qué se hace } \\
\text { en un huerto? }\end{array}$ & $\begin{array}{l}\text { (7) Las personas plantan } \\
\text { (8) Las personas cultivan } \\
\text { (9)hay herramientas/maquinaria } \\
\text { (10) Se riega } \\
\text { (11) Se recoge la cosecha. } \\
\end{array}$ & Modelo de buerto \\
\hline & 3 & $\begin{array}{l}\text { 4) ¿Qué es un } \\
\text { huerto ecológico? }\end{array}$ & $\begin{array}{l}\text { (12) Ecológico significa sano para el } \\
\text { cuerpo. } \\
\text { (13) Ecológico significa que solo hay sol y } \\
\text { agua (spray para matar bichos, ningún tipo } \\
\text { de spray) } \\
\text { (14) En un huerto hay personas que hacen } \\
\text { diferentes funciones }\end{array}$ & $\begin{array}{l}\text { Modelo de buerto } \\
\text { ecológico }\end{array}$ \\
\hline & 4 & 5) Recapitulación & $\begin{array}{l}\text { (15) Elemento agua } \\
\text { (16) Elemento semilla } \\
\text { (17) Elemento tierra } \\
\text { (18) Elemento sol }\end{array}$ & $\begin{array}{lr}\text { Modelo de } \\
\text { plantas } \\
\text { seres vivos }\end{array}$ \\
\hline
\end{tabular}


Tabla 2. Continuación.

\begin{tabular}{|c|c|c|c|c|}
\hline Asamblea & $\begin{array}{l}\mathbf{N}^{\circ} \text { Total } \\
\text { Esquemas }\end{array}$ & Secuencia & Esquemas de conocimiento & $\begin{array}{l}\text { Modelos } \\
\text { iniciales }\end{array}$ \\
\hline 2 & 3 & $\begin{array}{ll}\text { 6) Orden } & \text { de } \\
\text { importancia } & \text { de } \\
\text { los elementos del } \\
\text { huerto }\end{array}$ & $\begin{array}{l}\text { (19) Recoger es esencial } \\
\text { (20) Plantar y cultivar (como mismo } \\
\text { proceso) } \\
\text { (21) Cultivar es esencial (proceso } \\
\text { completo) } \\
\text { (22) Cada planta necesita su tiempo (hay } \\
\text { que esperar para recoger y no todas las } \\
\text { plantas tienen el mismo tiempo) }\end{array}$ & $\begin{array}{l}\text { Modelo de las } \\
\text { plantas como } \\
\text { seres vivos }\end{array}$ \\
\hline
\end{tabular}

Los esquemas que afloran muestran las ideas infantiles acerca de los distintos conceptos: por ejemplo, en la secuencia 4 de la asamblea 2 conectan el concepto de ecológico con la idea de que es sano para el cuerpo. Otro esquema es considerar que un huerto es ecológico cuando solo hay sol y agua. Esto supone un esquema con un nivel mayor de complejidad dado que es capaz de buscar una causa y no solo una consecuencia. Por otro lado, el hecho de que surja el esquema de usar spray y se discutan las condiciones para ello, se puede considerar un esquema más avanzado por las relaciones causa y efecto que niños y niñas son capaces de establecer. Los esquemas mentales no solo se conectan entre secuencias dentro de una misma asamblea, también entre asambleas realizadas. Así, en la secuencia 5 de la asamblea 2, cuando plantean un orden de importancia de los elementos del huerto; el elemento considerado más importante fue el agua. Este elemento lo relacionan mejor con las plantas, probablemente por un esquema de conocimiento surgido en la asamblea 1, las plantas necesitan algo de agua porque si no se secan, que se ha incorporado a esta asamblea. Esto podría evidenciar que durante las asambleas se construyen de manera activa conocimientos y que esta conexión entre asambleas permite dirigir el discurso científico hacia esquemas más avanzados, dado que en este caso aflora también la idea de incluir el sol como elemento importante en el huerto.

En la asamblea 3 se explicitaron un total de 13 esquemas (Tabla 3), uno de ellos aportado por el docente. En cada secuencia se observa un movimiento hacia esquemas más complejos, o más avanzados de conocimiento. Por ejemplo, en la secuencia 3, los esquemas avanzan hacia la idea de las plantas necesitan sol para convertirse en árboles. El modelo inicial que parece estar patente durante toda la asamblea es el del ciclo de vida de las plantas. Por otro lado, se aprecia que el discurso hace emerger esquemas con más matices, pasando de visiones extremistas tipo todo o nada a una visión más relativista, como ocurre en el esquema 11 de la secuencia 3, cada planta tiene su forma y su tiempo para crecer. El modelo inicial implícito en todos los casos parece ser el modelo del ciclo de vida de las plantas, complementario al modelo de planta como ser vivo, emergido en asambleas anteriores. Al final de la asamblea surgen además ciertos tecnicismos como polinización o néctar. 
Tabla 3. Esquemas y modelos explicativos emergentes identificados en las asambleas 3 y 4 (Fuente: Elaboración propia).

\begin{tabular}{|c|c|c|c|c|}
\hline Asamblea & $\begin{array}{l}\mathbf{N}^{\circ} \text { Total } \\
\text { Esquemas }\end{array}$ & Secuencia & Esquemas de conocimiento & $\begin{array}{l}\text { Modelos } \\
\text { iniciales }\end{array}$ \\
\hline \multirow[t]{3}{*}{3} & 4 & $\begin{array}{l}\text { 1)Experimento } \\
\text { con semillas }\end{array}$ & $\begin{array}{l}\text { (1) De las semillas salen las plantas } \\
\text { porque interviene el sol y la lluvia, los } \\
\text { cuidados de las personas y palabras } \\
\text { bonitas. } \\
\text { (2) Las plantas salen como flores. } \\
\text { (3) Las plantas son flores, pero salen } \\
\text { débiles y se hacen fuertes más tarde. } \\
\text { (4) Las plantas pueden ser árboles o } \\
\text { arbustos. }\end{array}$ & $\begin{array}{l}\text { Modelo de ciclo de } \\
\text { vida de las } \\
\text { plantas }\end{array}$ \\
\hline & 7 & $\begin{array}{l}\text { 3) ¿Por qué una } \\
\text { ha crecido y otra } \\
\text { no? }\end{array}$ & $\begin{array}{l}\text { (5) Las plantas crecen en su temporada. } \\
\text { (6) Las plantas necesitan tiempo para } \\
\text { crecer. } \\
\text { (7) Las plantas necesitan el sol para } \\
\text { convertirse en árboles. } \\
\text { (8) Las plantas necesitan espacio para } \\
\text { crecer. } \\
\text { (9) Para que las plantas crezcan hay que } \\
\text { decirles cosas bonitas. } \\
\text { (10) Las plantas necesitan paja para } \\
\text { crecer (inferido por docente). } \\
\text { (11) Cada planta tiene su forma, y su } \\
\text { tiempo para crecer. }\end{array}$ & $\begin{array}{l}\text { Modelo de ciclo de } \\
\text { vida de las } \\
\text { plantas }\end{array}$ \\
\hline & 2 & $\begin{array}{l}\text { 5) Conclusiones } \\
\text { finales }\end{array}$ & $\begin{array}{l}\text { (12) Las plantas no son flores (no todas } \\
\text { las plantas tienen flores). } \\
\text { (13) No todas las flores son iguales. }\end{array}$ & \\
\hline \multirow[t]{3}{*}{4} & 5 & $\begin{array}{l}\text { 1)Experimento } \\
\text { sobre } \\
\text { profundidad de la } \\
\text { semilla }\end{array}$ & $\begin{array}{l}\text { (1) La semilla necesita profundidad para } \\
\text { crecer. } \\
\text { (2) La semilla necesita estar en } \\
\text { superficie para salir (en profundidad se } \\
\text { ahoga por el agua). } \\
\text { (3) La semilla más profunda sale, pero } \\
\text { más tarde, en (profundidad necesita } \\
\text { tiempo). } \\
\text { (4) La semilla más profunda sale pero } \\
\text { necesita tiempo y fuerza. } \\
\text { (5) La semilla necesita aire. }\end{array}$ & $\begin{array}{lrr}\text { Modelo ciclo } & \text { de } \\
\text { vida de } & \text { las } \\
\text { plantas } & & \\
\text { Modelo } & & \text { de } \\
\text { plantas } & \text { como } \\
\text { seres vivos } & & \end{array}$ \\
\hline & 6 & $\begin{array}{l}\text { 2)Experimento } \\
\text { con algodón y } \\
\text { tierra }\end{array}$ & $\begin{array}{l}\text { (6) Retomado por el docente: las } \\
\text { semillas necesitan tierra y agua. } \\
\text { (7) Las semillas necesitan agua. } \\
\text { (8) La semilla nace en algodón. } \\
\text { (9) Las semillas necesitan luz (para } \\
\text { germinar). } \\
\text { (10) Creado por el docente: la semilla } \\
\text { nace en algodón. } \\
\text { (11) Las plantas no crecen en las nubes. }\end{array}$ & $\begin{array}{lll}\text { Modelo ciclo de } \\
\text { vida de las } \\
\text { plantas }\end{array}$ \\
\hline & 1 & $\begin{array}{l}\text { 3)Experimento } \\
\text { con agua y sin } \\
\text { agua }\end{array}$ & $\begin{array}{l}\text { (12) El arroz sale de una planta (se } \\
\text { planta una planta). }\end{array}$ & $\begin{array}{l}\text { Modelo de ciclo de } \\
\text { vida de una } \\
\text { planta }\end{array}$ \\
\hline
\end{tabular}


Tabla 3. Continuación

\begin{tabular}{|c|c|c|c|c|}
\hline Asamblea & $\begin{array}{l}\mathbf{N}^{\mathbf{o}} \text { Total } \\
\text { Esquemas }\end{array}$ & Secuencia & Esquemas de conocimiento & $\begin{array}{l}\text { Modelos } \\
\text { iniciales }\end{array}$ \\
\hline \multirow[t]{3}{*}{4} & 12 & $\begin{array}{l}\text { 4)Experimento } \\
\text { con luz y sin Luz }\end{array}$ & $\begin{array}{l}\text { (13) Las semillas no necesitan luz para } \\
\text { germinar. } \\
\text { (14) las plantas que no están verdes se } \\
\text { están muriendo. } \\
\text { (15) las plantas pueden tener color } \\
\text { blanco (no ser verdes). } \\
\text { (16) las plantas que son blancas son } \\
\text { viejas. } \\
\text { (17) las plantas a las que no le da el sol } \\
\text { son blancas / a las que da el sol son } \\
\text { verdes. } \\
\text { (18) una planta tapada se muere } \\
\text { las plantas necesitan luz para vivir (no } \\
\text { para germinar). } \\
\text { (20) las plantas necesitan un mínimo de } \\
\text { tierra para crecer verdes. } \\
\text { (21) la planta no crece igual si se tapa } \\
\text { (que si no se tapa). } \\
\text { (22) las plantas necesitan oxigeno para } \\
\text { vivir. } \\
\text { (23) las plantas que parecen enfermas } \\
\text { pueden sobrevivir. } \\
\text { (24) las plantas necesitan luz y agua para } \\
\text { vivir (no para germinar). }\end{array}$ & $\begin{array}{l}\text { Modelo de ciclo de } \\
\text { vida de una } \\
\text { planta } \\
\text { Modelo de planta } \\
\text { como seres vivos }\end{array}$ \\
\hline & 2 & $\begin{array}{l}\text { 5)Experimento } \\
\text { con tierra y sin } \\
\text { tierra }\end{array}$ & $\begin{array}{l}\text { (25) las semillas germinan en algodón } \\
\text { de azúcar. } \\
\text { (26) las plantas necesitan agua para } \\
\text { crecer. }\end{array}$ & $\begin{array}{lr}\text { Modelo } & d e \\
\text { plantas } & \text { como } \\
\text { seres vivos } & \end{array}$ \\
\hline & 1 & $\begin{array}{l}\text { 6)Experimento } \\
\text { profundidad }\end{array}$ & $\begin{array}{l}\text { (27) La semilla en profundidad necesita } \\
\text { fuerza para nacer. }\end{array}$ & $\begin{array}{l}\text { Modelo de ciclo de } \\
\text { vida de las } \\
\text { plantas }\end{array}$ \\
\hline
\end{tabular}

En la asamblea 4 se han identificado un total de 27 esquemas, que no aparecen de manera lineal a lo largo del discurso, sino que en muchas ocasiones los niños y niñas retoman, apoyan o aceptan dichos esquemas a lo largo del discurso. Cada secuencia se enfoca desde un experimento diferente a partir de un elemento clave para la germinación de las semillas y posterior crecimiento de la planta. De manera similar a la asamblea anterior, los esquemas avanzan hacia ideas más complejas, y en algún caso son retomados por el docente: por ejemplo, en la secuencia 2 aparece las semillas necesitan tierra y agua, lo que permite seguir avanzando en el discurso y aflorar otros esquemas. El experimento de comparar dos semillas, una con luz y otra sin luz, hace explicitar un mayor número de esquemas (12 en total), respecto al resto de secuencias. Durante la asamblea 4, se observa cómo surgen esquemas complejos de conocimiento, más abstractos, a medida que avanza el discurso, como las plantas necesitan oxigeno para vivir o bien, la diferenciación entre dos procesos complejos, para estas edades, como germinación y el crecimiento de las plantas, que se construyen en paralelo a lo largo de todo el discurso registrado. Los esquemas que se movilizan ganan en matices, apreciaciones y restricciones, como el esquema 24 las plantas necesitan luzy agua para vivir (no para germinar) lo cual denota el avance hacia un pensamiento más critico. El modelo inicial emergente que acompaña durante toda la asamblea es el modelo del ciclo de vida de las plantas, acompañando, en algunas ocasiones, al modelo de planta como ser vivo. 


\section{Conclusiones}

Un trabajo reciente muestra que, según los docentes en ejercicio, el principal problema a la hora de trabajar temas científicos en EI es la falta de formación científica, desde dos aspectos, disciplinar y didáctica, así como a la falta de recursos específicos de ciencias (Cantó y Serrano 2018). No obstante, y de acuerdo con estos autores, enseñar ciencias en EI pasa no solo por hacer experimentos y emplear recursos apropiados, sino sobre todo por fomentar estrategias de tipo cognitivo como el cuestionamiento, la duda, verbalizar las observaciones de los fenómenos que acontecen, así como propiciando el desarrollo más propios del "saber hacer" en ciencias como la observación, la formulación de hipótesis, o la emisión de conclusiones. La argumentación en ciencias se convierte así en una estrategia idónea para ello.

Nuestros datos muestran que los niños de EI, a través de la SD, participan en un discurso científico, aportando información simple o estructurada en esquemas, argumentando a favor o en contra de los esquemas de conocimiento que han emergido, estableciendo condiciones para ellos y haciendo emerger modelos iniciales de conocimiento. Los datos, en línea con otros estudios (Monteira y Jiménez-Aleixandre 2016; Franco y Munford 2018), han mostrado el nivel de minuciosidad en la elaboración de conocimiento al que los niños pueden llegar en la medida en que han sido involucrados en prácticas científicas reales y significativas en torno a su experiencia en el HEE. Por ello, advertimos la necesidad de que los docentes adquieran conciencia sobre el funcionamiento del discurso científico y que reconozcan y potencien las capacidades de los niños para participar en él, no solo en términos de indagación, sino también de argumentación y modelización, al nivel propio de la escuela infantil. Esa toma de conciencia debe incluir tanto el plano conceptual como el comunicativo y vincularse a prácticas auténticamente científicas, de forma que supere aproximaciones anecdóticas (Couso 2014). Creemos, siguiendo a Monteira, Jiménez-Aleixandre y Siry (2020), que una mayor seguridad de los docentes respecto de las capacidades de los niños menores de 6 años y del valor de su mediación y guía potencia el desarrollo de la competencia científica y puede encaminar el desarrollo de la alfabetización científica desde estas edades. En próximos estudios nos ocuparemos de la naturaleza y posibilidades de esa intervención docente.

\section{Agradecimientos}

Investigación financiada por Proyecto de Investigación Educativa desarrollado durante el curso 2017-2018, denominado "El Huerto Ecológico Escolar como contexto para el desarrollo de la competencia científica en la etapa de infantil. Un estudio de caso". (PIV-040/17) concedido por la Consejería de Educación de la Junta de Andalucía.

\section{Referencias bibliográficas}

Acevedo J.A, Vázquez A., Manassero M.A. (2003) Papel de la educación CTS en una alfabetización científica y tecnológica para todas las personas. Revista Electrónica de Enseñanza de las Ciencias, 2(2), 80-111.

Acevedo J.A. (2004) Reflexiones sobre las finalidades de la enseñanza de las ciencias: Educación científica para la ciudadanía. Revista Eureka sobre Enseñanza y Divulgación de las Ciencias, 1(1), 3-15.

Anderson J., Ellis J., Jones A. (2014) Understanding early elementary children's conceptual knowledge of plant structure and function through drawings. CBE Life Science Education, 13, 375-386.

Asencio-Cabot E.C. (2017) La educación científica: percepciones y retos actuales. Educación y Educadores, 20(2), 282-296. doi: 10.5294/edu.2017.20.2.7 
Cáceres J. (2012) El huerto escolar de hoy, la empresa sostenible del mañana. En I Congreso Virtual Internacional sobre Innovación Pedagógica y Praxis Educativa INNOVAGOGÍA, Sevilla.

Canedo-Ibarra S.P., Castelló-Escandell J., García-Wehrle P., Gómez-Galindo A., Morales-Blake A.R. (2012) Cambio conceptual y construcción de modelos científicos precursores en educación infantil. Revista Mexicana de Investigación Educativa, 17 (54), 691-727.

Cantó Doménech J., de Pro A., Solbes J. (2016) ¿Qué ciencias se enseñan y cómo se hace en las aulas de educación infantil? La visión de los maestros en formación inicial. Enseñanza de las Ciencias, 34.3, 25-50.

Cantó J., Serrano N. (2018) ¿Cuáles son los principales problemas para hacer presentes las ciencias en las aulas de educación infantil?: La visión de los maestros en ejercicio. Enseñanza de las Ciencias, No Extraordinario, 1995-2000.

COSCE (2011) Informe Enciende. Análisis, reflexiones y propuestas para un acercamiento de la ciencia al mundo escolar que promueva en los niños el interés por la ciencia, el aprendizaje cientifico y una visión no estereotipada de la empresa científica y sus protagonistas. Barcelona: Editorial Rubes.

Couso D. (2014) De la moda de "aprender indagando" a la indagación para modelizar: una reflexión crítica. En M.A. Herás, A. Lorca, B. Vázquez, A. Wamba, R. Jiménez. Investigación y transferencia para una educación en ciencias: Un reto emocionante (pp.1-28). Huelva: Servicio de Publicaciones Universidad de Huelva.

Domínguez G., Barrio L. (2001) Lenguaje, pensamiento y valores. Una mirada al aula. Madrid: Ediciones de la Torre.

Egea-Fernández J.M., Egea-Sánchez J.M., Guerrero, M. (2016) Huertos Escolares como recurso para la Educación Agroecológica. El caso de la región de Murcia. Agroecología, 11(1), 19-29.

Escutia M. (2009) El huerto escolar ecológico. Barcelona: Graó.

Eugenio M., Aragón L. (2016) Experiencias en torno al huertos ecológico como recurso didáctico y contexto educativo en la formación inicial de maestros/as. Revista Eureka sobre Enseñanza y Divulgación de las Ciencias, 13(3), 667-679. Recuperado de: http://hdl.handle.net/10498/18504

Franco L. G., Munford D. (2018) Aanálise de interações discursivas em aulas de ciências: ampliando perspectivas metodológicas na pesquisa em Argumentação. Educaşão em Revista, 34. doi: http://dx.doi.org/10.1590/0102-4698182956

García-González E., Schenetti M. (2019) Las escuelas al aire libre como contexto para el aprendizaje de las ciencias en infantil. El caso de la Scuola nel BoscoVilla Ghigi. Revista Eureka sobre Enseñanza y Divulgación de las Ciencias, 16(2), 2204. doi: 10.25267/Rev_Eureka_ensen_divulg_cienc.2019.v16.i2.2204

Garrido M. (2007) La evolución de las ideas de los niños sobre los seres vivos. (Tesis doctoral). Universidad da Coruña. Recuperado de: Repositorio da UDC (http://hdl.handle.net/2183/7330)

Greca I.M., Moreira M.A. (2000) Mental models, conceptual models, and modelling. International Journal of Science Education, 22(1), 1-11.

Grimm-Rice J. (2011) A case study: using a school garden to create Meaningful Learning. Unpublished Masters Thesis, Ohio, University. 
Gutiérrez J.M. (coord.) (1998) Ideas previas y Educación Ambiental. Bilbao: Fundación Bilbao Bizkaia Kutxa y Dep. de Educación, Universidades e Investigación del Gobierno Vasco. Centro de Experimentación Escolar de Pedernales-Sukarrieta. (Disponible en http://goo.gl/IVPv6a).

Hodson D. (1994) Hacia un enfoque más crítico del trabajo de laboratorio. Enseñanza de las Ciencias, 12, 299-313.

Jiménez-Aleixandre M.P., Bravo B., Puig B. (2009) ¿Cómo aprende el alumnado a usar y evaluar pruebas?. Aula de Innovación Educativa, 186, 10-15.

Jiménez-Aleixandre M.P., Crujeiras-Pérez B. (2017) Epistemic practices and Scientific practices in Science Education. En K.S. Taber y B. Akpan (Eds.). Science Education-an International Course Companion (pp. 69-80). The Netherlands: Sense Publishers.

Jiménez-Aleixandre M.P., Puig B. (2010) Argumentación y evaluación de explicaciones causales en ciencia: el caso de la inteligencia. Alambique: Didáctica de las ciencias experimentales, 63, 11-18.

Jorba J., Gómez I., Prat A. (Eds.) (2000) Hablary escribir para aprender. Uso de la lengua en situación de enseñanza-aprendizaje desde las áreas curriculares. Madrid: Síntesis/ICE UAB

Justi R., Gilbert J.K. (2002) Modelling teachers'views on the nature of modelling, and implications for the education of modellers. International Journal of Science Education, 24(4), 369-387.

Lemke J. (1997) Aprender a hablar ciencia: lenguaje, aprendizaje y valores. Barcelona: Paidós.

Llerena G., Espinet M. (2017) Agroecología escolar. Barcelona: Pollen Edicions.

Lorenzo Flores M., Sesto Varela V., García-Rodeja Gayoso, I. (2018) Una propuesta didáctica para la construcción de un modelo precursor del aire en la Educación Infantil. Ápice. Revista De Educación Cientifica, 2(2), 55-68. doi: https://doi.org/10.17979/arec.2018.2.2.4628

Marín N. (2005) La enseñanza de las ciencias en Educación Infantil. Granada: Grupo Editorial Universitario.

Márquez C., Bach J. (2007) Una propuesta de análisis de las representaciones de los alumnos sobre el ciclo del agua. Enseñanza de las Ciencias de la Tierra, 15.3, 280-286.

Mazas B., Gil-Quílez M.J., Martínez-Peña B., Hervás A. Muñoz A. (2018) Los niños de infantil piensan, actúan hablan sobre el comportamiento del aire y del agua. Enseñanza de las Ciencias, 36(1), 163-180.

McNair S., Stein M. (2001) Drawing on their understanding: using illustrations to invoke deeper thinking about plants. Paper presented at the Association for the Education of Teachers of Science Annual Meeting, held January 18-21, 2001, in Costa Mesa, CA.

Mercier H. (2011) Reasoning serves argumentation in children. Cognitive Development, 26(3), 177-191.

Mérida Serrano R., González Alfaya E., Olivares García M.A. (2017) Estrategias y modalidades de argumentación oral en las asambleas de Educación Infantil. Un estudio multicaso. Revista Complutense de Educación, 28(2), 445-462.

Migdalek M., Rosemberg C., Santibáñez C. (2015) La Génesis de la Argumentación. Un Estudio con Niños de 3 a 5 Años en Distintos Contextos de Juego. Íkala, Revista de Lenguaje y Cultura, 19. doi:10.17533/udea.ikala.v19n3a03. 
Monteira S.F., Jiménez Aleixandre M.P. (2016) The practice of using evidence in kindergarten: The role of purposeful observation. Journal of Research in Science Teaching 53(8), 12321258.

Monteira S.F., Jiménez Aleixandre M.P. (2019) ¿Cómo llega el agua a las nubes? Construcción de explicaciones sobre cambios de estado en educación infantil. Revista Eureka sobre Enseñanza y Divulgación de las Ciencias 16(2), 2101. doi: 10.25267/Rev_eureka_ensen_divulg_cienc.2019.v16i2.2101.

Monteira S.F., Jiménez-Aleixandre M.P., Siry C. (2020) Scaffolding Children's Production of Representations Along the Three Years of ECE: a Longitudinal Study. Research in Science Education. https://doi.org/10.1007/s11165-020-09931-z

Morillo Moronta I. (2008) Una nueva forma de enseñar ciencias en el contexto social. Laurus Revista de Educación, 14 (26), 337-318. Disponible en: http:/ / www.redalyc.org/articulo.oa?id=7611149105.

Palou J., Fons M. (2013) Investigar en un escenario social denominado aula. Cultura y Educación, 25 (4), 489-494.

Pedrinaci E., Caamaño A., Cañal P., Pro A. (2012) 11 ideas clave: El desarrollo de la competencia cientifica. Barcelona: Graó.

Pontes A., Poyato F.J., Oliva J.M. (2017) Concepciones sobre evaluación en la formación inicial del profesorado de ciencias, tecnología y matemáticas. Revista Iberoamericana de Evaluación Educativa, 9(1), 91-107.

Pujol R.M. (2003) Didáctica de las ciencias en la educación primaria. Madrid: Síntesis.

Ríos I. (2013) Aprender conceptos: el conocimiento científico del mundo. Textos de didáctica de la lengua y la literatura, 64, 23-31.

Rodríguez-Marín F., Fernández-Arroyo J., García J.E. (2015) El huerto escolar ecológico como herramienta para la educación en y para el decrecimiento. Investigación en la Escuela, 86, 35-48.

Ruiz Bikandi U. (2002) ¿Lengua oral formal en la educación infantil? Aula de Innovación Educativa, 111, 23-27.

Sánchez S. (2010) Interacción y didáctica de la lengua en educación infantil. En A. Costa, M.D. García, P. García, P. Ribera (Coord.) Interacció comunicativa i ensenyament de llengües, (pp. 203-209) España: Universidad de Valencia.

Sánchez S. (2016) La lengua oral: entre la familia y la escuela. En M. Fons, J. Palou (Coords.) Didáctica de la Lengua y la Literatura en Educación Infantil, (pp. 93-108). Madrid: Síntesis.

Sanmartí N. (2007) Hablar, leer y escribir para aprender ciencia. En P. Fernández (coordra.) La competencia en comunicación lingïística en las áreas del currículo. Colección Aulas de Verano. Madrid: MEC.

Tunnicliffe S.D. (2020) Emerging Biology in the Early Years: How young children learn about the living world. UK: Taylor \& Francis Group.

Tunnicliffe S.D., Reiss M.J. (2000) Building a model of the environment: how do children see plants? Journal of Biological Education, 34(4), 172-177.

Villanueva X. (2017) El aprendizaje de las plantas como seres vivos: una metodología basada en el dibujo infantil. IKASTORRATZA. E-Revista de Didáctica, 18, 106-123. doi: 10.37261/18_alea/6

Wells G. (1987) Apprenticeship in literacy. Interchange, 18(1-2), 109-123. 
Anexo 1. Secuencia de actividades

\begin{tabular}{|c|c|c|c|}
\hline Actividades & Intención didáctica & $\begin{array}{l}\text { Instrumento de } \\
\text { recogida de } \\
\text { información }\end{array}$ & Duración \\
\hline $\begin{array}{l}\text { Act.1. Primera asamblea: ¿Qué } \\
\text { sabemos del huerto ecológico? }\end{array}$ & $\begin{array}{l}\text { Explorar las ideas previas de los niños } \\
\text { en torno a los siguientes temas: qué y } \\
\text { cómo es un huerto, dónde hay huertos } \\
\text { y qué se hace en un huerto. }\end{array}$ & $\begin{array}{l}\text { Papel continuo } \\
\text { (mural) } \\
\text { Audio } \\
\text { Video } \\
\text { Cuaderno del } \\
\text { observador }\end{array}$ & 25 minutos \\
\hline $\begin{array}{l}\text { Act. 2. Dibujos individuales sobre } \\
\text { sus modelos iniciales de huerto y } \\
\text { explicación de sus dibujos. }\end{array}$ & $\begin{array}{l}\text { Explicitar los modelos iniciales de } \\
\text { huerto de los niños y explicar los } \\
\text { elementos presentes en él. }\end{array}$ & $\begin{array}{l}\text { Dibujos } \\
\text { individuales } \\
\text { Audio } \\
\text { Cuaderno del } \\
\text { observador }\end{array}$ & 45 minutos \\
\hline Act. 3. Notas a casa & $\begin{array}{l}\text { Escribir los niños a su nivel qué era } \\
\text { para él o para ella un huerto ecológico, } \\
\text { la respuesta debía ser transcrita por la } \\
\text { familia. }\end{array}$ & Nota a casa & $\begin{array}{l}\text { Una semana de } \\
\text { plazo }\end{array}$ \\
\hline $\begin{array}{l}\text { Act. 4. Segunda asamblea: ¿qué } \\
\text { elementos hay en un huerto? } \\
\text { Ordenamos según prioridad. }\end{array}$ & $\begin{array}{l}\text { Proponer por el alumnado los } \\
\text { elementos relativos al huerto y } \\
\text { ordenara esos elementos en función de } \\
\text { la importancia que tienen dentro del } \\
\text { huerto. }\end{array}$ & $\begin{array}{l}\text { Papel continuo } \\
\text { Audio } \\
\text { Vídeo } \\
\text { Cuaderno del } \\
\text { observador }\end{array}$ & 45 minutos \\
\hline $\begin{array}{l}\text { Act. } 5 \text { ¿Cómo será nuestro } \\
\text { huerto? }\end{array}$ & $\begin{array}{l}\text { Visualizar distintos tipos de huertos } \\
\text { para analizar diferentes opciones y } \\
\text { plantear la posibilidad de hacer un } \\
\text { huerto en el cole y argumentar cuál era } \\
\text { el más idóneo }\end{array}$ & $\begin{array}{l}\text { Cuaderno del } \\
\text { observador }\end{array}$ & 45 minutos \\
\hline $\begin{array}{l}\text { Act. } 6 \text { ¿Dónde ubicamos el } \\
\text { huerto? }\end{array}$ & $\begin{array}{l}\text { Conectar con sus ideas previas sobre } \\
\text { los elementos presentes en el huerto y } \\
\text { su orden de importancia. Considerar el } \\
\text { sol, la luz, como elementos principal y } \\
\text { decidir dónde ubicar el huerto en el } \\
\text { patio del centro. }\end{array}$ & Papel continuo & $\begin{array}{l}2 \text { sesiones de } \\
45 \text { minutos }\end{array}$ \\
\hline $\begin{array}{l}\text { Act. } 7 \text {. Experimentos en torno a } \\
\text { elementos del huerto. Para un } \\
\text { mismo tipo de semilla a) se } \\
\text { sembró a distinta profundidad; b) } \\
\text { con tierra y sin tierra; c) con tierra } \\
\text { y una sin agua y con agua; y d) } \\
\text { con tierra y una sin luz y con luz. }\end{array}$ & $\begin{array}{l}\text { Comprobar algunas hipótesis } \\
\text { formuladas por el alumnado sobre el } \\
\text { orden de importancia de los elementos } \\
\text { del huerto. }\end{array}$ & $\begin{array}{l}\text { Cuaderno del } \\
\text { observador. } \\
\text { Audio. }\end{array}$ & 45 minutos \\
\hline $\begin{array}{l}\text { Act. 8. Semilleros y plantas (I). } \\
\text { Extracción y observación de } \\
\text { semillas de distintas verduras. } \\
\text { Siembra de semillas diferentes en } \\
\text { semilleros. Etiquetado y } \\
\text { colocación en el espacio } \\
\text { habilitado como invernadero. }\end{array}$ & $\begin{array}{l}\text { Construir conocimiento en base a sus } \\
\text { ideas previas sobre los elementos } \\
\text { considerados como prioritarios por el } \\
\text { alumnado: Las semillas y las plantas. }\end{array}$ & $\begin{array}{l}\text { Registro } \\
\text { fotográfico. } \\
\text { Cuaderno del } \\
\text { observador. }\end{array}$ & 1 hora \\
\hline $\begin{array}{l}\text { Act. 9. Semilleros y plantas (II). } \\
\text { Observación de las partes de una } \\
\text { planta, descripción e } \\
\text { identificación de los plantones. }\end{array}$ & $\begin{array}{l}\text { Construir conocimiento en base a sus } \\
\text { ideas previas sobre los elementos } \\
\text { considerados como prioritarios por el } \\
\text { alumnado: Las semillas y las plantas. }\end{array}$ & $\begin{array}{l}\text { Audio } \\
\text { Vídeo } \\
\text { Cuaderno del } \\
\text { observador. }\end{array}$ & 1 hora \\
\hline
\end{tabular}


Anexo 1. Continuación

\begin{tabular}{|c|c|c|c|}
\hline Actividades & Intención didáctica & $\begin{array}{l}\text { Instrumento de } \\
\text { recogida de } \\
\text { información }\end{array}$ & Duración \\
\hline Act. 10. Sembrando en el bancal. & $\begin{array}{l}\text { Preparación del bancal para sembrar } \\
\text { usando principios basados en } \\
\text { permacultura. }\end{array}$ & Vídeo & 3 horas \\
\hline $\begin{array}{l}\text { Act. 11. Tercera asamblea: ¿qué le } \\
\text { ha ocurrido a las semillas? }\end{array}$ & $\begin{array}{l}\text { Argumentar en base a los resultados } \\
\text { obtenidos de la actividad 8: siembra de } \\
\text { distintos tipos de semillas con } \\
\text { velocidad de germinación diferente }\end{array}$ & $\begin{array}{l}\text { Audio } \\
\text { Vídeo } \\
\text { Cuaderno del } \\
\text { observador }\end{array}$ & 22 minutos \\
\hline $\begin{array}{l}\text { Act. 12. Cuarta asamblea: ¿qué } \\
\text { resultados hemos obtenido de } \\
\text { nuestros experimentos? }\end{array}$ & $\begin{array}{l}\text { Argumentar en base a los resultados } \\
\text { obtenidos de los experimentos } \\
\text { realizados en la actividad } 7 .\end{array}$ & $\begin{array}{l}\text { Audio } \\
\text { Video } \\
\text { Cuaderno del } \\
\text { observador } \\
\end{array}$ & 41 minutos \\
\hline $\begin{array}{l}\text { Act. } 13 \text {. Ampliando el huerto: } \\
\text { huerto vertical. }\end{array}$ & $\begin{array}{l}\text { Reutilizar materiales para crear un } \\
\text { huerto vertical para aumentar la } \\
\text { biodiversidad y fomentar la fauna } \\
\text { auxiliar. }\end{array}$ & $\begin{array}{l}\text { Registro } \\
\text { fotográfico }\end{array}$ & 1 hora \\
\hline $\begin{array}{l}\text { Act. 14. Observando y } \\
\text { explorando en el huerto a través } \\
\text { de lupas, visores de insecto y } \\
\text { pinzas. }\end{array}$ & $\begin{array}{l}\text { Contribuir al desarrollo de la } \\
\text { competencia científica favoreciendo la } \\
\text { observación y exploración de las } \\
\text { plantas e insectos presentes en el } \\
\text { huerto. Manejar material destinado a la } \\
\text { observación. }\end{array}$ & & $\begin{array}{l}\text { Tiempo de } \\
\text { recreos }(30 \\
\text { minutos) }\end{array}$ \\
\hline $\begin{array}{l}\text { Act. } 15 . \text { Nos organizamos para el } \\
\text { riego: creación de un calendario } \\
\text { de riego. }\end{array}$ & $\begin{array}{l}\text { Considerar la importancia del agua } \\
\text { para las plantas del huerto. Fomentar el } \\
\text { trabajo cooperativo para organizar las } \\
\text { labores de cuidado y riego del bancal y } \\
\text { huerto vertical }\end{array}$ & Papel continuo & $\begin{array}{l}\text { Sesiones diarias } \\
\text { de } 15-20 \\
\text { minutos }\end{array}$ \\
\hline $\begin{array}{l}\text { Act. 16. Salida a la Casa de los } \\
\text { Colores (Diputación de Cádiz) }\end{array}$ & $\begin{array}{l}\text { Conocer la procedencia de algunas de } \\
\text { las semillas y plantones del huerto. } \\
\text { Realizar talleres complementarios a los } \\
\text { contenidos trabajados en torno al } \\
\text { huerto: insectos, alimentación } \\
\text { saludable y jardinería. }\end{array}$ & & 5 horas \\
\hline $\begin{array}{l}\text { Act. } 17 . \text { Recogiendo la cosecha: } \\
\text { taller de alimentación. }\end{array}$ & $\begin{array}{l}\text { Recolectar plantas del huerto para } \\
\text { consumo propio. Preparar una } \\
\text { ensalada con distintos tipos de } \\
\text { lechugas ecológicas recogidas del } \\
\text { bancal. }\end{array}$ & & 1 hora \\
\hline $\begin{array}{l}\text { Act. 18. Elaboración de una ficha } \\
\text { individual de los pasos seguidos } \\
\text { para preparar la ensalada. }\end{array}$ & $\begin{array}{l}\text { Recordar y repasar el proceso realizado } \\
\text { para asimilar y reforzar hábitos de } \\
\text { consumo e higiene. }\end{array}$ & Ficha & 45 minutos \\
\hline $\begin{array}{l}\text { Act. 19. Dibujos individuales del } \\
\text { modelo de huerto. }\end{array}$ & $\begin{array}{l}\text { Evaluar sus modelos finales de huerto } \\
\text { al finalizar la secuencia didáctica. }\end{array}$ & $\begin{array}{l}\text { Dibujos } \\
\text { Audios } \\
\text { Cuaderno del } \\
\text { observa }\end{array}$ & 1 hora \\
\hline
\end{tabular}

